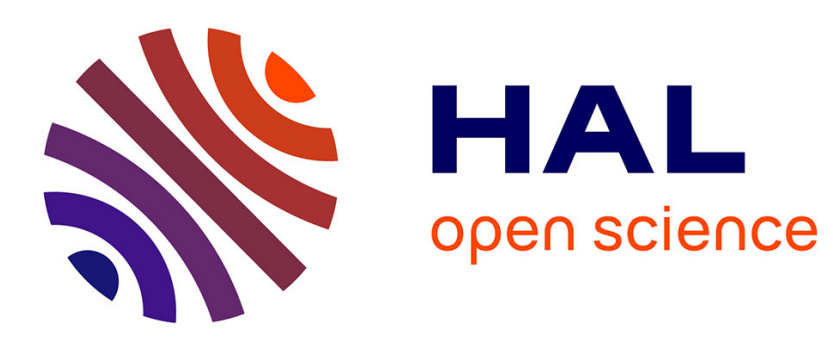

\title{
Détermination précise des paramètres cristallins au voisinage du changement de phase cubique quadratique dans RbCaf3
}

\author{
C. Ridou, M Rousseau, A. Freund
}

\section{- To cite this version:}

C. Ridou, M Rousseau, A. Freund. Détermination précise des paramètres cristallins au voisinage du changement de phase cubique quadratique dans RbCaf3. Journal de Physique Lettres, 1977, 38 (17), pp.359-363. 10.1051/jphyslet:019770038017035900 . jpa-00231396

\section{HAL Id: jpa-00231396 https://hal.science/jpa-00231396}

Submitted on 1 Jan 1977

HAL is a multi-disciplinary open access archive for the deposit and dissemination of scientific research documents, whether they are published or not. The documents may come from teaching and research institutions in France or abroad, or from public or private research centers.
L'archive ouverte pluridisciplinaire HAL, est destinée au dépôt et à la diffusion de documents scientifiques de niveau recherche, publiés ou non, émanant des établissements d'enseignement et de recherche français ou étrangers, des laboratoires publics ou privés. 


\title{
DÉTERMINATION PRÉCISE DES PARAMÈTRES CRISTALLINS AU VOISINAGE DU CHANGeMeNT DE PHASE CUBIQUE QUADRATIQUE DANS $\mathrm{RbCaF}_{3}$
}

\author{
C. RIDOU, M. ROUSSEAU \\ Laboratoire de Physique de l'Etat Condensé (*) et Faculté des Sciences, 72017 Le Mans Cedex, France
}

\section{A. FREUND}

Institut Max Von Laüe-Paul-Langevin 156 X, 38042 Grenoble, Cedex, France

(Reçu le 5 juillet 1977, accepté le 29 juillet 1977)

\begin{abstract}
Résumé. - Nous avons effectué une détermination précise des paramètres cristallins de $\mathrm{RbCaF}_{3}$ sur un diffractomètre rayons $X$ à deux axes. Nous avons mesuré une discontinuité de $10^{-3} \AA$, ce qui met en évidence un faible caractère du premier ordre pour la transition cubique-quadratique dans $\mathrm{RbCaF}_{3}\left(\mathrm{O}_{\mathrm{h}}^{1} \rightarrow \mathrm{D}_{4 \mathrm{~h}}^{18}\right)$. Le profil assymétrique de la raie cubique 004 près de la température de transition $T_{\mathrm{c}}$ s'interprète par la présence de microdomaines quadratiques.
\end{abstract}

\begin{abstract}
The temperature dependence of the lattice constant of $\mathrm{RbCaF}_{3}$ has been accurately measured using a double-crystal X-ray diffraction technique. A discontinuity of $10^{-3} \AA$ has been measured and one can conclude that the character of the cubic to tetragonal phase transition in $\mathrm{RbCaF}_{3}\left(\mathrm{O}_{\mathrm{h}}^{1} \rightarrow \mathrm{D}_{4 \mathrm{~h}}^{18}\right)$ is of weak first order. The assymetric profile of the cubic 004 line near $T_{\mathrm{c}}$ is interpreted in terms of tetragonal microdomains.
\end{abstract}

1. Introduction. - La transition cubique-quadratique dans la pérovskite fluorée $\mathrm{RbCaF}_{3}$ a été mise en évidence et étudiée par différentes techniques : diffractométrie rayons $\mathrm{X}$ sur poudre et diffusion Raman [1], diffusion inélastique de neutrons [2], RPE [3]. Tous ces résultats montrent que la quadratisation $\left(\mathrm{O}_{\mathrm{h}}^{1} \rightarrow \mathrm{D}_{4 \mathrm{~h}}^{18}\right)$ est dûe à la condensation d'un mode mou de symétrie $R_{25}$. Comme pour les transitions de même nature observées dans $\mathrm{KMnF}_{3}$ et $\mathrm{SrTiO}_{3}$, le paramètre d'ordre est l'angle de rotation $\varphi$ des octaèdres $\mathrm{CaF}_{6}$ autour des axes pseudocubiques $\langle 001\rangle$. En accord avec le modèle de Alefeld [4], les mesures faites sur poudre montrent bien une diminution du paramètre $a$ dans la phase quadratique en fonction de la température. Par contre l'augmentation du paramètre $c$ ne peut s'expliquer que si l'on tient compte d'un couplage du paramètre d'ordre avec la contrainte induite par la déformation [5]. D'autre part, les mesures de Shirane et al. [6] font apparaître une légère discontinuité dans l'intensité de la diffusion élastique au point $R$ de la zone de Brillouin cubique pour $\mathrm{KMnF}_{3}$ alors que la variation en fonction de la température semble continue pour

(*) Equipe de Recherche Associée au C.N.R.S. no 682.
$\mathrm{SrTiO}_{3}$. On peut donc considérer que la transition est faiblement du premier ordre dans $\mathrm{KMnF}_{3}$ alors qu'elle serait purement du second ordre dans $\mathrm{SrTiO}_{3}$. Pour $\mathrm{RbCaF}_{3}$, les mesures que nous avons effectuées sur poudre ne sont pas suffisamment précises pour conclure sur l'ordre de la transition. Cependant les mesures de diffusion élastique des neutrons au point $\mathbf{R}$ mettent en évidence une variation brutale de l'intensité diffusée au passage de la transition [7]. Pour tenter d'élucider ce problème, il devenait indispensable comme dans $\mathrm{SrTiO}_{3}$ [8] d'augmenter la précision des mesures du paramètre cristallin au voisinage de la transition. Pour cela nous avons adapté un cryostat rayons $\mathrm{X}$ sur un diffractomètre deux axes mis au point par A. Freund [9] à l'Institut LaueLangevin à Grenoble.

Ce travail comprend une partie expérimentale décrivant les différentes méthodes utilisées et les résultats obtenus, puis une discussion sur l'apport de ces techniques de mesures fines de paramètres cristallins dans l'étude d'un changement de phase.

2. Méthodes expérimentales et résultats. -2.1 ECHANTILLON ET CRYOGÉNIE. - Nous avons utilisé un monocristal de $\mathrm{RbCaF}_{3}$ préparé par la méthode de Bridgman-Stockbarger [10]. Une fois taillé, le cristal 
se présente sous la forme d'un parallélépipède rectangle de dimensions

$$
7,25 \times 6,04 \times 5,41 \mathrm{~mm}^{3}
$$

dont la plus grande face est perpendiculaire à un axe quaternaire [001].

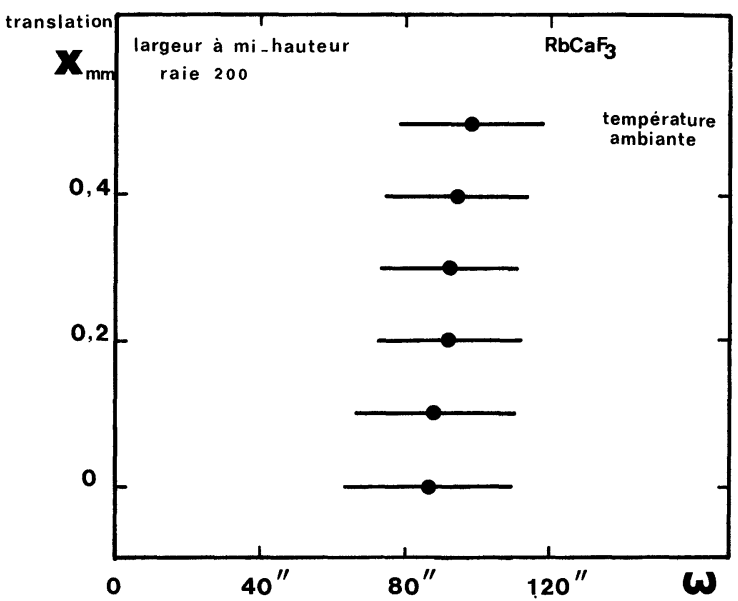

Fig. 1. - Etude de la mosaïcité dans $\mathrm{RbCaF}_{3}$ en fonction de la translation $x$ du cristal ; $\omega$ représente l'angle de rotation du cristal autour de la position de Bragg pour les plans réticulaires (002).

[Crystal translation dependence of the mosaic structure in $\mathrm{RbCaF}_{3}$; $\omega$ is the crystal rotation angle through the 002 reflection Bragg angle.]

La mosaïcité du cristal mesurée sur un diffractomètre à rayons $\gamma$ [9] est de 40" environ (Fig. 1). Elle ne varie pratiquement pas en fonction de la translation du cristal, i. e. de l'endroit dans le cristal où elle est mesurée. La position $x=0$ du cristal correspond à une valeur de translation pour laquelle la moitié du faisceau $\gamma$ de $0,2 \mathrm{~mm}$ de largeur pénètre dans le cristal. La légère courbure des plans réflecteurs visible sur la figure 1 est très petite par rapport à la largeur. Son influence sur les mesures aux rayons $\mathbf{X}$ est négligeable. Les spectres enregistrés montrent des profils parfaitement symétriques. Ceci est important pour la précision de la détermination du paramètre de la maille.

Toutes nos mesures de paramètre cristallin en fonction de la température ont été effectuées en plaçant le cristal à l'intérieur d'un cryostat à circulation d'azote liquide muni de fenêtres en béryllium. Un régulateur type MB 4000 (licence Anvar) permet de stabiliser la température à mieux que le 1/100 degré. Nous avons adapté ce cryostat sur l'axe de rotation d'un goniomètre horizontal Philips PW 1380 modifié qui constitue le deuxième axe du diffractomètre à deux axes.

2.2 Diffractométrie SIMPLE CRISTAL. - Nous avons effectué des mesures absolues du paramètre cristallin [11] sur un diffractomètre rayons $X$ à un axe, i.e. celui de l'échantillon et du détecteur. Nous avons orienté un monocristal de $\mathrm{RbCaF}_{3}$ en position de réflexion pour les plans réticulaires (004). A partir des enregistrements en $\theta-2 \theta$ de la raie de diffraction 004, nous mesurons les angles de diffraction $\theta_{1}$ et $\theta_{2}$ associés aux positions de réflexion (1) et (2) du cristal définies sur la figure 2. L'angle de Bragg $\theta_{\mathrm{B}}$ est alors égal à :

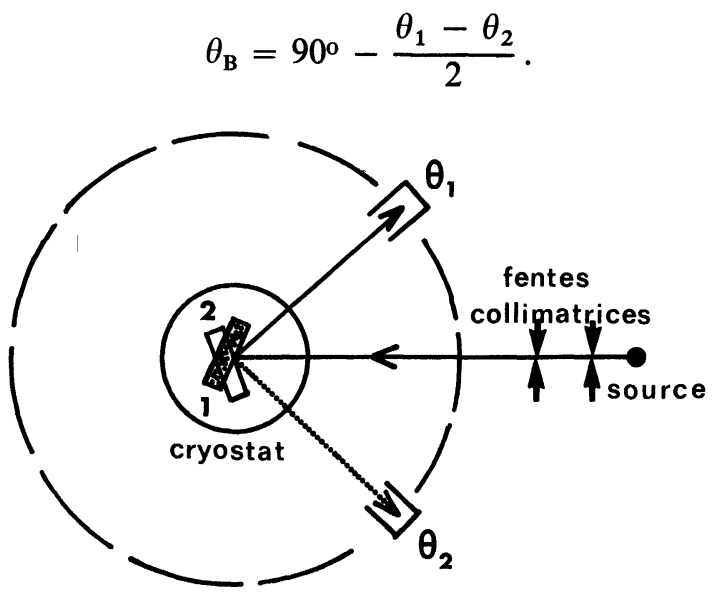

FIG. 2. - Schéma du diffractomètre rayons $\mathrm{X}$ montrant les positions symétriques du cristal pour la mesure du paramètre cristallin par la méthode de Bond modifiée.

[Schematic set-up of the X-ray diffractometer showing the symmetrical crystal positions for measuring lattice constants with a modined Bond technique.]

Nous éliminons ainsi l'erreur de zéro de l'appareil et obtenons une mesure absolue du paramètre de la maille. Cette mesure doit être corrigée de l'effet de la réfraction. Pour cela, nous avons effectué une modification de la méthode de Bond en mesurant l'angle du cristal et non l'angle du détecteur. La largeur à mi-hauteur mesurée pour les raies 004 est de 0,12 degré (soit $7^{\prime}$ ) en $\theta$ provenant de la largeur spectrale du rayonnement $\mathbf{X}$ et de la divergence du faisceau d'une part, et de la mosaïcité de l'échantillon d'autre part. L'ensemble des erreurs dues à l'imprécision du diffractomètre, à la statistique de comptage et à l'alignement vertical de l'échantillon est inférieur à $1 \%$ de la largeur mesurée. La précision du paramètre est :

$$
\frac{\Delta a}{a}=\operatorname{cotg} \theta \Delta \theta=2,2 \times 10^{-5}
$$

pour la réflexion 004 et le rayonnement $\mathrm{CuK}_{\alpha_{1}}$ $(\lambda=1,54056 \AA)$ et :

$$
\frac{\Delta a}{a}=7,8 \times 10^{-6}
$$

pour la raie 004 et le rayonnement $\mathrm{CrK}_{\beta_{1}}$ $(\lambda=2,0848 \AA)$.

Nous avons suivi le déplacement de la raie 004 de $200 \mathrm{~K}$ à $140 \mathrm{~K}$ à partir des spectres de diffraction enregistrés pour les positions (1) et (2) du cristal défi- 
nies sur la figure 2. De $200 \mathrm{~K}$ à $194 \mathrm{~K}$, les spectres obtenus ont une raie unique correspondant à la diffraction par les plans réticulaires (004) à partir desquels nous mesurons l'angle de Bragg cubique $\theta_{\mathbf{B}}$. Nous avons trouvé :

$$
\theta_{\mathrm{B}}=69,587 \pm 0,001 \text { degré à } 200,6 \mathrm{~K} \text {. }
$$

Cet angle diminue légèrement en fonction de la température jusqu'à la transition, ce qui correspond à une faible contraction de la maille cristalline dans la phase cubique. Le coefficient de dilatation linéaire correspondant est égal à :

$$
\frac{\Delta a_{\mathrm{c}}}{\Delta T}=7 \times 10^{-5} \AA \mathrm{K}^{-1} .
$$

De $194 \mathrm{~K}$ à $193 \mathrm{~K}$, pendant le changement de phase, la raie 004 s'élargit brutalement. Elle présente une structure non résolue qui impose l'utilisation de la méthode du double-cristal. En dessous de $193 \mathrm{~K}$, dans la phase quadratique, les spectres enregistrés comprennent deux raies. La plus intense correspond à un angle de Bragg inférieur à celui mesuré dans la phase cubique. Les mesures faites sur poudre ayant montré que le paramètre $c$ est supérieur au paramètre $a$, cette raie est dûe à la diffraction par les plans réticulaires (004). Elle permet de calculer le paramètre quadratique $c$. L'autre raie dont l'angle de Bragg est supérieur, est associé aux plans réticulaires (400), (040). Elle permet de calculer le paramètre quadratique $a$. L'intensité de la raie 400, 040 étant faible par rapport à celle de la raie 004 , on peut en conclure que, dans la phase quadratique, le cristal est presque monodomaine. Nous avons également observé que l'écart angulaire entre la raie 004 d'une part, et la raie 400, 040 d'autre part n'est pas le même pour les positions (1) et (2) d'enregistrement. Cette anomalie peut s'expliquer par la présence de domaines quadratiques légèrement désorientés par rapport aux axes cristallographiques de la maille cubique. Sur la figure 3 nous avons porté le résultat de nos mesures des paramètres cubique et quadratique à partir de spectres complètement résolus.

2.3 DifFRACTOMÉTRIE DOUBLE-CRISTAL. - Le diffractomètre utilisé comprend deux axes de rotation [9]. L'axe A est celui d'une table goniométrique sur laquelle nous avons placé un premier cristal de $\mathrm{RbCaF}_{3}$; l'axe $B$ est celui du goniomètre horizontal Philips sur lequel nous avons adapté le cryostat. Le cristal A dont la mosaïcité est inférieure à celle du cristal $B$ est utilisé comme monochromateur avant. Sur la figure 4 nous présentons le schéma de l'appareil en position d'enregistrement. Cet arrangement symétrique $n_{1}-n$ des deux cristaux permet d'éliminer tout élargissement de la raie provenant de la largeur spectrale et de la divergence horizontale du faisceau $X$. Ainsi nous avons pu réduire la largeur à mi-hauteur à 0,05 degré. Ceci nous a permis de mesurer la position angulaire

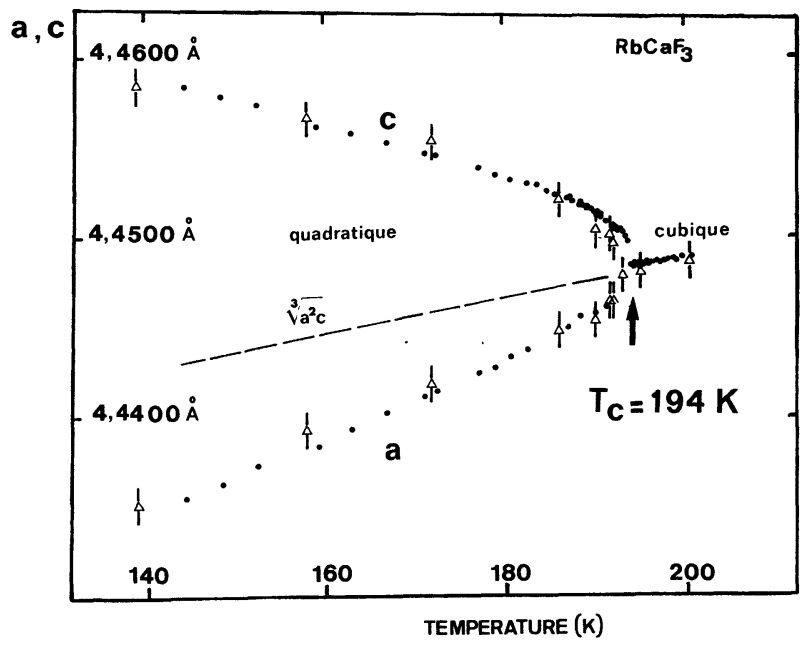

FIG. 3. - Variation des paramètres cristallins de $\mathrm{RbCaF}_{3}$ de $140 \mathrm{~K}$ à $200 \mathrm{~K}$. Les points noirs représentent nos mesures sur monocristal par la méthode de Bond. Les triangles blancs correspondent à nos précédentes mesures sur poudre.

[Lattice constants in $\mathrm{RbCaF}_{3}$ from $140 \mathrm{~K}$ to $200 \mathrm{~K}$. The dashed circles represent our experimental results obtained with a single crystal Bond technique. The open triangles correspond to our previous measurements on powder.]

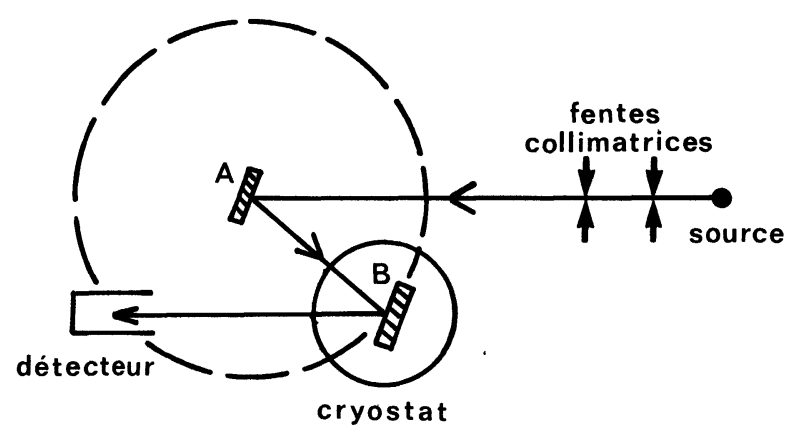

Fig. 4. - Schéma du diffractomètre deux axes rayons $\mathrm{X}$ en position $\mathrm{n}_{1}-\mathrm{n}$ des deux cristaux.

[Schematic set-up of the double-crystal X-ray diffractometer showing the $n_{1}-n$ arrangement of the two crystals.]

de la raie 004 à mieux que 0,0005 degré compte tenu du fait que la précision relative dụ diffractomètre est supérieure à la précision absolue. Pour le rayonnement $\mathrm{CrK}_{\beta_{1}}$ on obtient :

$$
\frac{\Delta a}{a}=3,2 \times 10^{-6}
$$

pour une variation relative du paramètre.

Nous avons effectué à la température ambiante un enregistrement en $\theta-2 \theta$ de la raie 004. Le profil obtenu est symétrique. Il peut être ajusté avec une lorentzienne dont les caractéristiques sont données sur la figure 5. Par contre, à 194,5 K, nous observons (Fig. 6) une dissymétrie assez marquée sur le flanc gauche de la raie (angles de Bragg décroissants). Nous avons ajusté de façon satisfaisante le profil en superposant deux lorentziennes : l'une fine et intense 


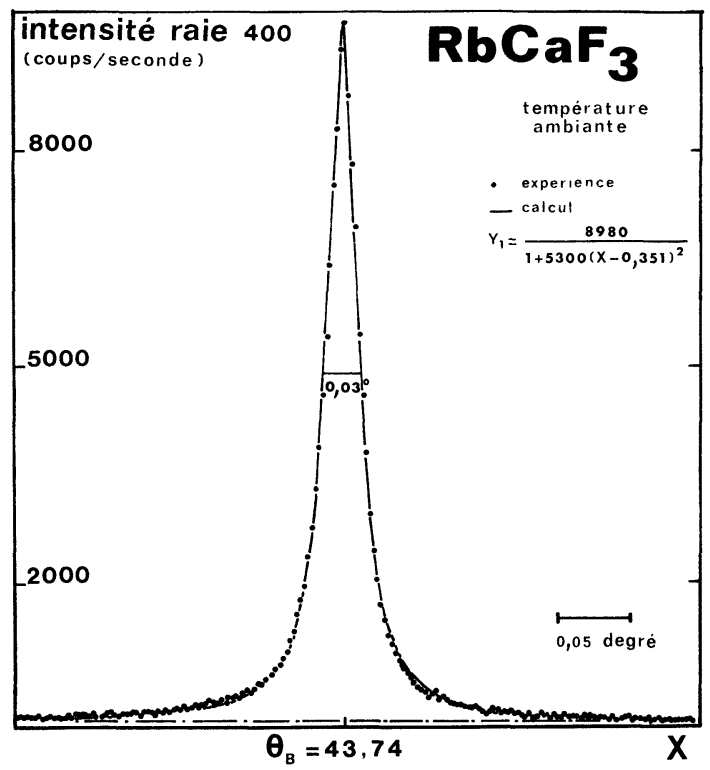

FIG. 5. - Profil symétrique de la raie 004 de $\mathrm{RbCaF}_{3}$ à température ambiante pour le rayonnement $\mathrm{CuK}_{\alpha_{1}}$.

[Symmetrical 004 profile of $\mathrm{RbCaF}_{3}$ recorded with a $\mathrm{CuK}_{\alpha_{1}}$ radiation at room temperature.]

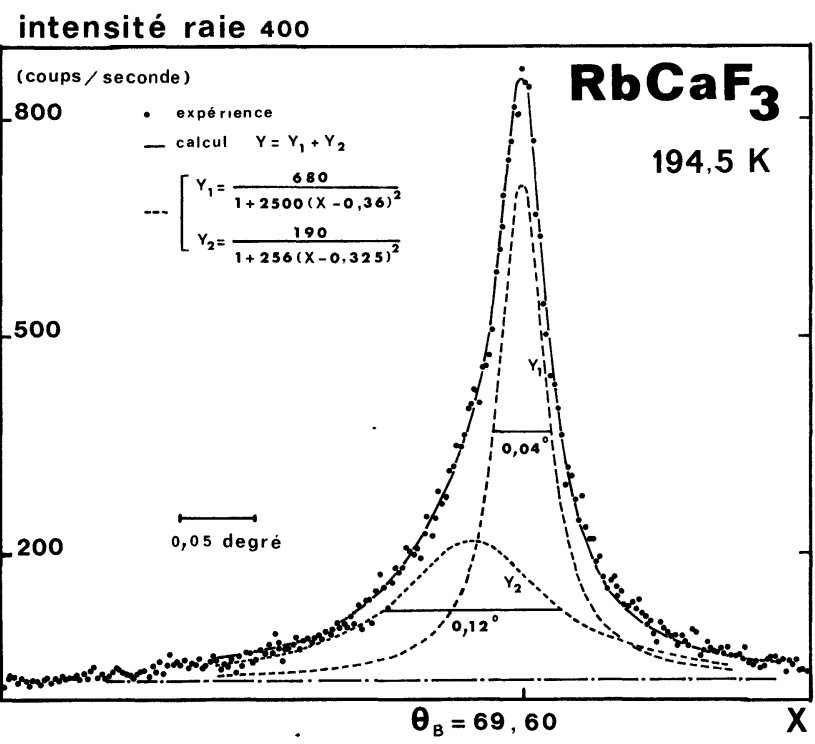

Fig. 6. - Profil assymétrique de la raie 004 de $\mathrm{RbCaF}_{3}$ à $194,5 \mathrm{~K}$ pour le rayonnement $\mathrm{CrK}_{\boldsymbol{\beta}_{1}}$,

[Unsymmetrical 004 profile of $\mathrm{RbCaF}_{3}$ recorded with a $\mathrm{CrK}_{\beta_{1}}$ radiation at $194.5 \mathrm{~K}$.]

correspond à la raie cubique non déformée, l'autre est large et faible. L'écart angulaire calculé entre les positions des sommets des deux lorentziennes est égal à 0,035 degré. De 194,14 K à 193,68 K les spectres enregistrés présentent une raie centrée sur l'angle de Bragg cubique. Sur le flanc gauche de celle-ci (angles de Bragg décroissants) apparaît progressivement la raie quadratique 004 en même temps que la raie cubique disparaît.
3. Discontinuité du paramètre cristallin à la transition. - L'hypothèse d'un gradient vertical de température dans le cristal permet d'expliquer la superposition des phases quadratique et cubique au passage de la transition. A partir de l'ajustement obtenu pour la raie 004 dans la phase cubique à $194,5 \mathrm{~K}$ nous avons superposé deux profils identiques en faisant varier les hauteurs au sommet. La figure 7 montre une

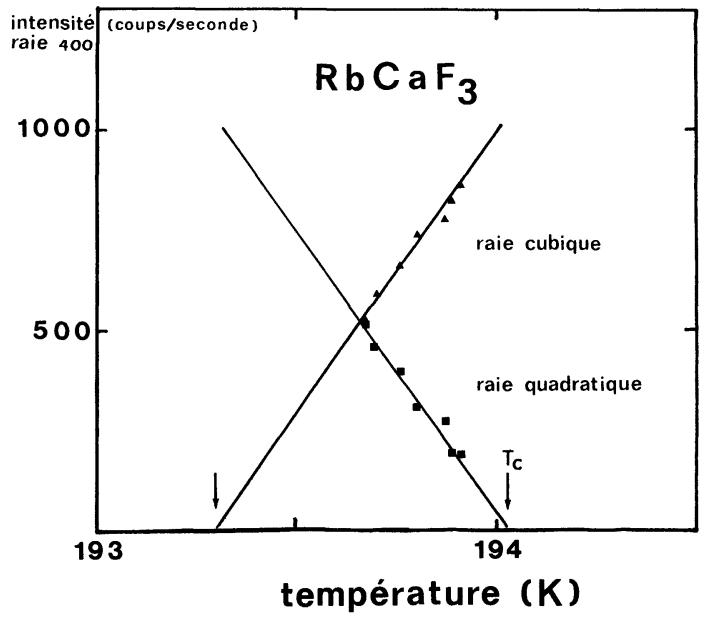

Fig. 7. - Evolution des intensités des raies cubique et quadratique au passage de la transition. L'écart entre les deux flèches mesure le gradient de température dans le cristal.

[Temperature dependence of the cubic and quadratic lines intensity through the phase transition. The variation between the two arrows measures the temperature gradient in the crystal.]

évolution linéaire, en sens inverse, des intensités des raies cubique et quadratique. Ce résultat vérifie bien l'hypothèse du gradient de température dans le cristal que l'on trouve égal à $0,7 \mathrm{~K}$ pour une hauteur de cristal de $7 \mathrm{~mm}$. La température de transition mesurée est $T_{\mathrm{c}}=194 \pm 1 \mathrm{~K}$. Elle diffère assez peu de celle trouvée précédemment sur poudre et en diffusion élastique des neutrons $\left(T_{\mathrm{c}}=193 \pm 1 \mathrm{~K}\right)$.

Nous représentons sur la figure 8 la variation de l'écart angulaire entre la raie cubique et la raie qua-

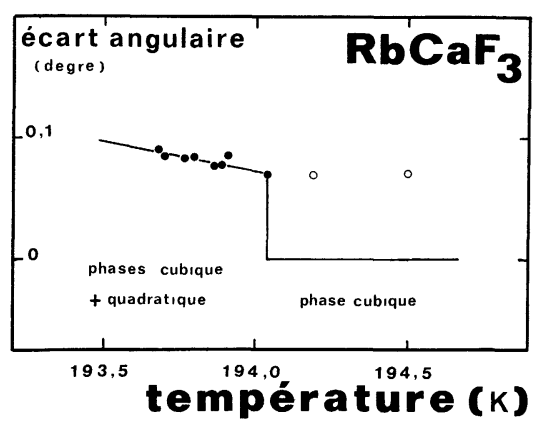

Fig. 8. - Variation de l'écart angulaire entre la raie cubique et la raie quadratique de 193,5 K à 194,5 K. Les ronds blancs correspondent à la position de la raie satellite dans la phase cubique.

[Temperature dependence of the angular variation between the cubic line and the quadratic line from 193.5 K to $194.5 \mathrm{~K}$. The open circles correspond to the cubic satellite line position.] 
dratique en fonction de la température. L'apparition brutale de la raie quadratique permet de conclure à un caractère du premier ordre pour cette transition. Nous mesurons une discontinuité du paramètre cristallin de $10,1 \times 10^{-4} \AA$ ce qui correspond à un angle de rotation des octaèdres de fluor de 0,86 degré. Cette mise en évidence d'un caractère faiblement du premier ordre pour la transition est en bon accord avec les expériences de biréfringence de Bates et al. [12].

On doit également remarquer que la raie quadratique 004 apparaît à l'endroit où se trouve, dans la phase cubique, la raie satellite du profil assymétrique étudié à 194,5 K (Fig. 5). On peut donc penser que la déformation de la raie cubique près de la transition est due à des fluctuations partiellement corrélées. En effet, les mesures de constantes élastiques effectuées dans ce composé par propagation d'ondes ultrasonores [13] et par diffusion Brillouin [14] montrent que les effets dûs aux fluctuations du paramètre d'ordre sont observés dans une large plage de température au-dessus de $T_{\mathrm{c}}$. D'après Levanyuck et Sannikov [15], ces fluctuations peuvent être responsables d'une discontinuité du paramètre d'ordre quand elles existent dans un large domaine de température et sont fortement couplées avèc les contraintes induites par la déformation. Dans cette hypothèse la longueur de corrélation calculée par la raie satellite à partir de la formule de Scherrer est de l'ordre de $2000 \AA$ (soit 450 mailles) à $0,5 \mathrm{~K}$ de la transition. Nous envisageons d'effectuer l'étude de l'évolution de la longueur de corrélation en fonction de la température afin de confirmer ou non cette hypothèse.

En ce qui concerne la superposition des phases cubique et quadratique au voisinage de $T_{\mathrm{c}}$ que nous avons attribuée qualitativement à un gradient de température, il est intéressant de noter qu'un effet analogue a été observé récemment par R. P. E. [16]. Il semble donc nécessaire de poursuivre ces études afin de différencier les causes extrinsèques (gradient de température) des causes intrinsèques comme la présence de microdomaines de symétrie quadratique dans la phase cubique. A cet effet, nous effectuons actuellement des expériences de RMN dans ce composé.

Remerciements. - Nous remercions M. J. Y. Gesland du laboratoire de Physique de l'Etat Condensé de la Faculté des Sciences du Mans pour la qualité des cristaux qu'il nous a préparés, ainsi que $\mathbf{M}$. Hustache de l'Institut Laue-Langevin de Grenoble pour son efficace collaboration technique.

\section{Bibliographie}

[1] Ridou, C., Rousseau, M., Gesland, J. Y. et Nouet, J., Ferroelectrics 12 (1976) 199.

Rushworth, A. J. et Ryan, J. F., Solid State Commun. 18 (1976) 1239.

[2] Kamitakahara, W. A. et Rotter, C. A., Solid State Commun. 17 (1975) 1350.

Rousseau, M., Nouet, J. et Almairac, R., à paraître.

Almairac, R., Rousseau, M., Gesland, J. Y., Nouet, J. et HeNNION, B., à paraître.

[3] Modine, F. A., Sonder, E., UnRuh, W. P., Finch, C. B. et Westbrook, R. D., Phys. Rev. B 10 (1974) 1623.

Rousseau, J. J., Rousseau, M. et Fayet, J. C., Phys. Status Solidi (b) 73 (1976) 625.

[4] Alefeld, B., Z. Phys. 222 (1969) 155.

[5] Slonczewski, J. C. et Thomas, H., Phys. Rev. B 1 (1970) 3599.

[6] Shirane, G., Minkiewicz, V. J. et Linz, A., Solid State Commun. 8 (1960) 1941.
[7] Rousseau, M., Nouet, J., Almairac, R. et Hennion, B., J. Physique Lett. 37 (1976) L-33.

[8] OKaSaki, A. et Kawaminami, M., Ferroelectrics 2 (1974) 91.

[9] Freund, A. et SCHNeider, J., J. Cryst. Growth 13/14 (1972) 247.

[10] GesLand, J. Y., Thèse, Le Mans (1977).

[11] Bond, W. L., Acta Cryst. 13 (1960) 814.

[12] Bates, J. B., MaJor, R. W. et Modine, F. A., Solid State Commun. 17 (1975) 1347.

[13] Ridou, C., Thèse, Le Mans (1977).

[14] Berger, J., Hauret, G. et Rousseau, M., à paraître.

[15] Levanyuk, A. P. et Sannikov, D. G., Usp. Fiz. Nauk 112 (1974) 561.

[16] Buzare, J. Y. et Fayet, J. C., Solid State Commun. 21 (1977) 1097.

Buzare, J. Y., Rousseau, J. J. et Fayet, J. C., Communication privée, à paraître. 